(C) The Author(s)

DOI: $10.1142 / \mathrm{S} 201019451860114 \mathrm{X}$

\title{
Measurement of the enhancement of the nuclear interaction yield with crystalline targets at cyclotron energies
}

\author{
E. Bagli \\ INFN Sezione di Ferrara, Via Saragat 1 \\ 44122 Ferrara, Italy \\ bagli@fe.infn.it \\ D. De Salvador \\ Dipartimento di Fisica e Astronomia, Università di Padova, Via Marzolo 8 \\ 35131 Padova, Italy \\ INFN Laboratori Nazionali di Legnaro, Viale dell'Università 2 \\ 35020 Legnaro, Italy \\ davide.desalvador@unipd.it \\ G. Guidi \\ COMECER Group spa, Via Maestri Del Lavoro 90 \\ 48014 Castel Bolognese, Italy \\ gguidi@comecer.com
}

Published 10 July 2018

\begin{abstract}
Ordered structures such as crystalline materials may help to enhance the nuclear interaction yield. Indeed, the aligned atoms act as a single entity on impinging charged particles, causing the trajectory to pass from its random motion to a deterministic one. In fact, Monte Carlo simulations suggested that specific crystal alignments allow for the enhancement of the production rate of nuclear inelastic reactions, because particles are forced to pass by the atomic nuclei more frequently than would happen in an amorphous material. Recent measurements we carried out at the AN2000 accelerator of the National Institute for Nuclear Physics Legnaro National Laboratories showed the experimental evidence of such an effect. A $643.5 \mathrm{keV}$ collimated proton beam was used to induce the ${ }^{18} \mathrm{O}(\mathrm{p}, \alpha)^{15} \mathrm{~N}$ reaction in an $\mathrm{Al}_{2} \mathrm{O}_{3}$ substrate oriented along the $<0001>$ axis. The capability of manipulating such an effect paves the way to studying innovative targets for the enhancement of the nuclear interaction yield with a constant density.
\end{abstract}

Keywords: Nuclear interactions; channeling.

PACS numbers: 25.55.Ci, 61.85.+p

\section{Introduction}

A fundamental part of the process for producing a radioisotope via cyclotron is the fabrication of a suitable target. Using an amorphous target allows for the production of a fixed number of radionuclides as a function of the beam energy. Such a technique proved to be reliable and is currently used all over the world. Charged particles that hit an

This is an Open Access article published by World Scientific Publishing Company. It is distributed under the terms of the Creative Commons Attribution 4.0 (CC-BY) License. Further distribution of this work is permitted, provided the original work is properly cited. 
amorphous target suffer random scatters from nuclei and electrons. On the contrary, charged particles interacting with a crystalline material are subject to coherent interactions, thanks to the periodicity of the crystalline atoms in the structure. ${ }^{1}$ Coherent interactions modify particle trajectories inside a medium, causing the variation of the interaction rate with atomic nuclei. Indeed, the correlated interaction leads particles to follow a preferential pathway in the crystal. Because of this, such effects can be exploited to modify the probability of a close encounter with nuclei in order to enhance or decrease the radioisotope production yield. Although such effect was experimentally observed at hundreds of $\mathrm{GeV}$ energies, ${ }^{2}$ there is a lack of experimental measurements at $\mathrm{MeV}$ energies, which are far more interesting with regard to their useful applications. In this paper, we present the measurement of anti-channeling for the enhancement of the radioisotope production yield.

\section{Channeling}

Channeling manifests itself when a charged particle impinges a crystal nearly aligned with the crystal plane or axes, ${ }^{1}$ i.e., when the angle between the particle direction and the crystal plane is lower than the critical angle for channeling $\theta_{c}$. Under channeling conditions, the particle is captured by the potential well formed by two neighboring planes and is forced to bounce between them. The critical angle depends on the crystal potential well depth $U$ and the particle momentum-velocity $p \beta, \theta_{c}=(U / p \beta)^{1 / 2}$. A particle under channeling can traverse a longer distance with respect to an amorphous medium for equal material density. Indeed, the particle interacts less frequently with nuclei and core electrons, losing energy only because of the interaction with valence electrons. The reduction of the interaction frequency with nuclei induces a lowering of the rate of nuclear inelastic interactions in a crystal. ${ }^{2}$

An interesting case is particles that enter the crystal at an angle close to the critical angle for channeling. Indeed, such particles repeatedly cross the crystal planes, impinging close to atomic nuclei. The constrained trajectory forces the particle to increase the probability of interaction with nuclei, over the probability exhibited by an amorphous target. In contrast, a particle that enters parallel to the crystalline planes bounces between them, in a region without nuclei. Therefore, its probability of interaction with nuclei decreases.

\section{Monte Carlo}

In order to quantify the deflection efficiency of the channeling process in bent crystals, Monte Carlo simulations were carried out using the Geant 4 toolkit. ${ }^{3}$ The channeling process is implemented by including DYNECHARM $++{ }^{4}$ and ECHARM ${ }^{5}$ into the Geant 4 channeling package. ${ }^{6}$ DYNECHARM++ allows the tracking of a relativistic charged particle inside a crystalline medium via the numerical integration of the classical equations of motion. The continuum potential approximation proposed by Lindhard is used. ${ }^{1}$ ECHARM allows the computation of the crystal's electrical characteristics within this approximation. 
The Geant4 application was developed on top of the 10.3 version of the toolkit, which allows for crystalline structures. ${ }^{7}$ All of the physical phenomena occurring for a channeled particle are strongly affected by the encountered number of nuclei and electrons, which depends on the particle trajectory. ${ }^{1}$ Therefore, the probability of a physics process occurring in the simulation has been weighted as a function of the density of the material experienced by a channeled particle. Such dependence on the probability of interaction allows for the correct evaluation of the deflection efficiency, which strongly depends on the particle charge sign. ${ }^{8}$ The simulations produced with Geant 4 were successfully compared with experimental data for both positive and negative particles under planar channeling ${ }^{8}$ and positive particles under axial channeling for two different crystal structure orientations. ${ }^{9}$

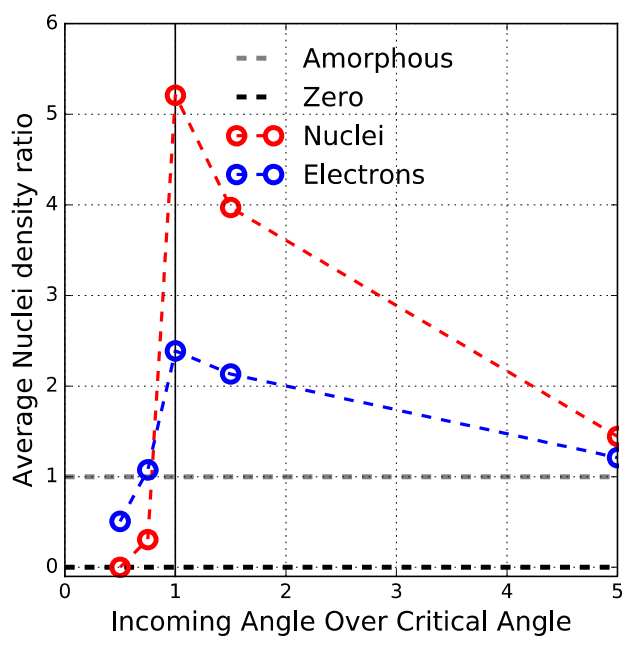

Fig. 1. Geant4 simulation of the modification of the nuclear interaction rate as a function of the particle incoming angle interacting with a $\mathrm{Si}(220)$ crystal. The particles impinge at $0.48 \mathrm{~A}$ from the $\mathrm{Si}(220)$ planes.

In order to quantify the probability of interaction with nuclei in a crystal, let us define the average density $X$ seen by a particle as the integral of the density of nuclei over the particle trajectory. Such a definition takes into account how the density varies as a function of the particle trajectory. Figure 1 (above) shows the evolution of $X$ as a function of the incoming angle depth into a Si (220) crystal for a channeled particle. A channeled particle will always stay far from the nuclei. Therefore, the probability of interaction with nuclei goes down approximately to zero and remains constant. On the contrary, a particle which crosses the crystal plane at an angle close to the critical channeling angle spans a range of densities, from close to zero to a nuclei density around 10 times higher than average. However, the time spent on the first and the second part of the trajectory is 
different. Indeed, because of the steep electromagnetic field in the rise, the particle will spend more time in the zone with high nuclei density, while it will go faster in the other zone. Therefore, the average density weighted over the particle trajectory becomes greater than one, approaching two at maximum. At last, when the angle with respect to the crystal plane is too big, $X$ tends to 1 , i.e., the crystal acts as an amorphous target. A similar enhancement could be observed in the transition between axial and planar channeling. In this case, the particle trajectory is forced to cross the region between the axis and the plane if the angle is similar to the critical angle for axial channeling. Such effect was observed with the Rutherford back-scattering technique and a similar behavior is expected for nuclear interactions.

\section{Experimental Measurements}

The accelerator used for the test was the AN2000 at the National Institute for Nuclear Physics Legnaro National Laboratories (INFN-LNL), a compact electrostatic-type accelerator with a maximum voltage terminal of $2 \mathrm{MeV}$. The limited output energy available from an accelerator of this size makes it an ideal facility to promote nuclear reaction events within the first layers of target materials.

At the RBS-Channeling line of AN2000, a high-resolution goniometer is installed with an angular resolution of $0.01^{\circ}$ and a minimum pressure inside the chamber of approximately $10^{-7}$ mbar. The goniometer allows the alignment of the crystal axis with respect to the incoming beam within the critical channeling angle $\theta_{c}$, i.e., the maximum misalignment angle for which channeling is visible. Indeed, $\theta_{c}$ for a proton at the maximum energy of $2 \mathrm{MeV}$ is of the order of $0.2^{\circ}$.

The system was equipped with three detectors. The first detector is a silicon detector for Rutherford back-scattering analysis (RBS). It was placed at $110 \mathrm{~mm}$ and at angle of $160^{\circ}$ from the target. The surface of the detector is $25 \mathrm{~mm}^{2}$. The second detector is a silicon detector for the nuclear resonance analysis (NRA) and was placed at $150^{\circ}$ and at a distance of $40 \mathrm{~mm}$ from the target. The detector has a $300 \mathrm{~mm}^{2}$ surface and was covered with a Mylar foil to prevent back-scattered particles from entering the detector. The third detector is a NaI scintillator with a surface of about $100 \mathrm{~cm}^{2}$. The detector was placed in a recess outside the vacuum chamber at a distance of $100 \mathrm{~mm}$.

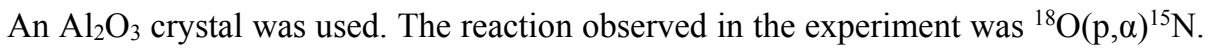
Oxygen has a $0.2 \%$ natural abundance of the ${ }^{18} \mathrm{O}$ isotope. The resonance at approximately $643.5 \mathrm{keV}$ was used. Figure 2 shows the results for a random orientation (Random), the axial orientation (Axial), and the transition between axial and planar orientation ${ }^{10}$ (Increase). A hint of the anti-channeling effect is clearly visible, since the nuclear interaction rate grows in a particular orientation. 


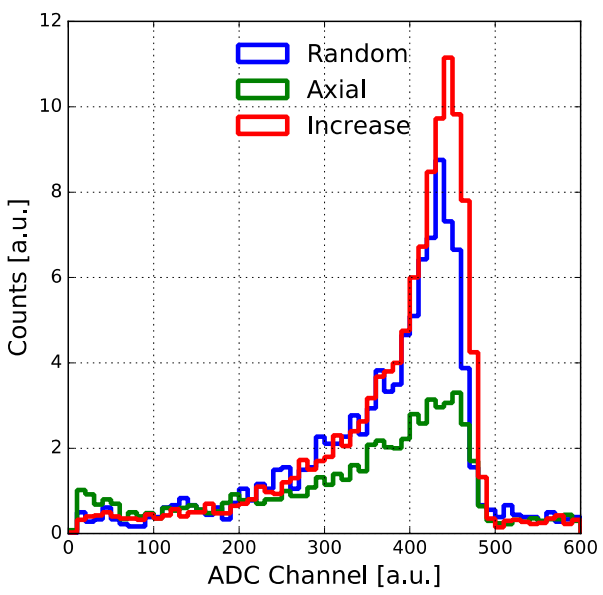

Fig. 2. Experimental measurements of the $\alpha$ particles generated in the reaction ${ }^{18} \mathrm{O}(\mathrm{p}, \alpha){ }^{15} \mathrm{~N}$ for a $643.5 \mathrm{keV}$ beam impinging on a $\mathrm{Al}_{2} \mathrm{O}_{3}$ crystal for random orientation (Random), axial orientation (Axial), and transition between axial and planar orientation (Increase).

\section{Conclusions}

Anti-channeling has been theoretically predicted and observed experimentally. Further studies are needed to prove that the condition is obtained for the specific predicted angles in order to use them for applications. The development of a crystalline target would allow for the usage of such crystals without modifying existing cyclotron nozzles. Indeed, the crystal can be oriented before usage and easily placed inside.

\section{Acknowledgments}

We acknowledge partial support by the National Institute of Nuclear Physics under the GECO (GEant4 Crystal Objects) project. We acknowledge the CINECA (Interuniversity Consortium High Performance Systems) award under the ISCRA (Italian SuperComputing Resource Allocation) initiative, for the availability of high-performance computing resources and support.

\section{References}

1. J. Lindhard, Danske Vid. Selsk. Mat. Fys. Medd. 34, 14 (1965).

2. W. Scandale et al., Nucl. Instrum. Meth. Phys. Res. Sect. B 268, 2655 (2010).

3. J. Allison et al., Nucl. Instrum. Meth. Phys. Res. Sect. A 835, 186 (2016).

4. E. Bagli and V. Guidi, Nucl. Instrum. Meth. Phys. Res. Sect. B 309, 124 (2013).

5. E. Bagli, V. Guidi, and V. A. Maisheev, Phys. Rev. E 81, 026708 (2010).

6. E. Bagli et al., Eur. Phys. J. C 74, 2996 (2014).

7. E. Bagli et al., Nucl. Instrum. Meth. Phys. Res. Sect. B 402, 304 (2017).

8. E. Bagli et al., Eur. Phys. J. C 77, 71 (2017).

9. W. Scandale et al., Phys. Letters B 760, 826 (2016).

10. A. Carnera et al., Phys. Rev. B 18, 995 (1978). 